\title{
Detection of interstellar HCS and its metastable isomer HSC: new pieces in the puzzle of sulfur chemistry ${ }^{\star}$
}

\author{
M. Agúndez ${ }^{1}$, N. Marcelino ${ }^{1}$, J. Cernicharo ${ }^{1}$, and M. Tafalla ${ }^{2}$ \\ ${ }^{1}$ Instituto de Física Fundamental, CSIC, C/ Serrano 123, 28006 Madrid, Spain \\ e-mail: marcelino.agundez@csic.es \\ ${ }^{2}$ Observatorio Astronómico Nacional (OAN), C/ Alfonso XII 3, 28014 Madrid, Spain
}

Received 31 January 2018 / Accepted 26 February 2018

\section{ABSTRACT}

\begin{abstract}
We present the first identification in interstellar space of the thioformyl radical (HCS) and its metastable isomer HSC. These species were detected toward the molecular cloud L483 through observations carried out with the IRAM $30 \mathrm{~m}$ telescope in the $\lambda 3 \mathrm{~mm}$ band. We derive beam-averaged column densities of $7 \times 10^{12} \mathrm{~cm}^{-2}$ for HCS and $1.8 \times 10^{11} \mathrm{~cm}^{-2}$ for HSC, which translate into fractional abundances relative to $\mathrm{H}_{2}$ of $2 \times 10^{-10}$ and $6 \times 10^{-12}$, respectively. Although the amount of sulfur locked by these radicals is low, their detection allows placing interesting constraints on the chemistry of sulfur in dark clouds. Interestingly, the $\mathrm{H}_{2} \mathrm{CS} / \mathrm{HCS}$ abundance ratio is found to be quite low, $\sim 1$, in contrast with the oxygen analog case, in which the $\mathrm{H}_{2} \mathrm{CO} / \mathrm{HCO}$ abundance ratio is around 10 in dark clouds. Moreover, the radical HCS is found to be more abundant than its oxygen analog, HCO. The metastable species HOC, the oxygen analog of HSC, has not yet been observed in space. These observational constraints are compared with the outcome of a recent model of the chemistry of sulfur in dark clouds. The model underestimates the fractional abundance of HCS by at least one order of magnitude, overestimates the $\mathrm{H}_{2} \mathrm{CS} / \mathrm{HCS}$ abundance ratio, and does not provide an abundance prediction for the metastable isomer HSC. These observations should prompt a revision of the chemistry of sulfur in interstellar clouds.
\end{abstract}

Key words. astrochemistry - line: identification - ISM: clouds - ISM: molecules - radio lines: ISM

\section{Introduction}

The chemistry of sulfur in interstellar clouds has attracted considerable attention from astronomers in the past decades. For example, defining the main reservoir of sulfur in cold dark clouds continues to be an important open question. A variety of simple sulfur-bearing species is observed in the gas phase of these environments. These comprise the hydride $\mathrm{H}_{2} \mathrm{~S}$, the carbon-bearing molecules CS, $\mathrm{H}_{2} \mathrm{CS}, \mathrm{C}_{2} \mathrm{~S}$, and $\mathrm{C}_{3} \mathrm{~S}$, the oxides $\mathrm{SO}$ and $\mathrm{SO}_{2}$, OCS, the nitrogen-bearing species NS, HNCS, and HSCN, and the ion $\mathrm{HCS}^{+}$(Adande et al. 2010; Agúndez \& Wakelam 2013). However, these species only account for less than $0.1 \%$ of the cosmic abundance of sulfur. Moreover, chemical models of cold dark clouds usually need to assume that sulfur is depleted in the gas phase by at least two orders of magnitude to reproduce the abundances derived from observations (Agúndez \& Wakelam 2013). However, in photodissociation regions such as the Horsehead Nebula, it is found that sulfur is not severely depleted, only by a factor of 3-4 (Goicoechea et al. 2006). The missing sulfur in cold dark clouds is commonly thought to be deposited onto dust grains, although it is not clear in which form. Some sulfur could be trapped in the core of grains as refractory compounds, such as FeS or MgS, although it is not known how much sulfur could be in this form. Unfortunately, the depletion factor of sulfur in diffuse interstellar clouds is uncertain because the $\mathrm{S}^{+}$lines are often saturated (Jenkins 2009). Ices could also be an important reservoir of sulfur in cold dark clouds. Solid OCS and $\mathrm{SO}_{2}$ are thought to be responsible for absorption features in the infrared spectra of young stellar objects, with inferred

\footnotetext{
${ }^{\star}$ Based on observations carried out with the IRAM $30 \mathrm{~m}$ Telescope. IRAM is supported by INSU/CNRS (France), MPG (Germany) and IGN (Spain).
}

abundances of $0.2-0.5 \%$ and $0.6-6 \%$, respectively, of the available sulfur (Palumbo et al. 1997; Boogert et al. 1997). It has been proposed that other ices such as polysulfanes could be a major reservoir of sulfur (Jiménez-Escobar \& Muñoz Caro 2011; Druard \& Wakelam 2012). It is also possible that gas-phase molecules that have not yet been identified could account for a significant fraction of this element.

The missing sulfur problem has attracted renewed attention in recent years. New potentially important sulfur reservoirs have been explored. For example, the $\mathrm{S}_{2}$-containing molecules $\mathrm{H}_{2} \mathrm{~S}_{2}, \mathrm{HS}_{2}$, and $\mathrm{S}_{2}$ have been unsuccessfully searched for toward the low-mass protostar IRAS 16293-2422 by Martín-Doménech et al. (2016), while the detection of gasphase $\mathrm{H}_{2} \mathrm{~S}$ in the shocked region L1157-B1 by Holdship et al. (2016) led the authors to conclude that a significant fraction of sulfur must be in the form of $\mathrm{H}_{2} \mathrm{~S}$ ice. The chemistry of sulfur in dark clouds has recently been revisited observationally by Fuente et al. (2016a) and theoretically by Vidal et al. (2017). These latter authors point to gas-phase atomic sulfur or $\mathrm{SH}$ and $\mathrm{H}_{2} \mathrm{~S}$ ices as the main sulfur reservoirs. It is also worth noting that two new sulfur-bearing molecules have recently been detected in space: $\mathrm{HS}_{2}$ has been identified in the Horsehead Nebula (Fuente et al. 2016b), and the ion $\mathrm{NS}^{+}$has been ubiquitously found in dense molecular clouds (Cernicharo et al. 2018).

In this Letter we present the first detection in interstellar space of two new sulfur-bearing molecules: the radical HCS, and its metastable isomer HSC. These species have been identified in the dense cloud L483, a source that hosts a rich chemistry, where we have recently discovered new molecules such as the ketenyl radical $(\mathrm{HCCO})$, protonated cyanogen $\left(\mathrm{NCCNH}^{+}\right)$, and the ion $\mathrm{NS}^{+}$(Agúndez et al. 2015a,b; Cernicharo et al. 2018). 
Table 1. Observed line parameters of HCS and HSC in L483.

\begin{tabular}{|c|c|c|c|c|c|c|c|c|}
\hline Species & Transition & $\begin{array}{c}\text { Frequency } \\
(\mathrm{MHz})\end{array}$ & $\begin{array}{c}E_{u} \\
(\mathrm{~K})\end{array}$ & $\begin{array}{c}A_{u l} \\
\left(\mathrm{~s}^{-1}\right)\end{array}$ & $g_{u}$ & $\begin{array}{c}V_{\mathrm{LSR}} \\
\left(\mathrm{km} \mathrm{s}^{-1}\right)\end{array}$ & $\begin{array}{c}\Delta v \\
\left(\mathrm{~km} \mathrm{~s}^{-1}\right)\end{array}$ & $\begin{array}{c}\int T_{A}^{*} \mathrm{~d} v \\
\left(\mathrm{mK} \mathrm{km} \mathrm{s}{ }^{-1}\right)\end{array}$ \\
\hline \multirow[t]{5}{*}{$\mathrm{HCS}$} & $2_{0,2}-1_{0,1} \quad J=5 / 2-3 / 2 \quad F=3-2$ & 80553.516 & 5.8 & $4.50 \times 10^{-7}$ & 7 & $+5.43(3)$ & $0.61(5)$ & $10.5(9)$ \\
\hline & $2_{0,2}-1_{0,1} \quad J=5 / 2-3 / 2 \quad F=2-1$ & 80565.596 & 5.8 & $4.34 \times 10^{-7}$ & 5 & $+5.33(5)$ & $0.77(12)$ & $10.4(12)$ \\
\hline & $2_{0,2}-1_{0,1} \quad J=3 / 2-1 / 2 \quad F=2-1$ & 80596.409 & 5.8 & $3.41 \times 10^{-7}$ & 5 & $+5.56(6)$ & $0.88(12)$ & $9.0(11)$ \\
\hline & $2_{0,2}-1_{0,1} \quad J=3 / 2-1 / 2 \quad F=1-0$ & 80611.994 & 5.8 & $2.51 \times 10^{-7}$ & 3 & $+5.32(10)$ & $0.70(20)$ & $3.5(11)$ \\
\hline & $2_{0,2}-1_{0,1} \quad J=3 / 2-1 / 2 \quad F=1-1$ & 80618.820 & 5.8 & $1.79 \times 10^{-7}$ & 3 & $+5.67(7)$ & $0.36(23)$ & $2.4(10)$ \\
\hline \multirow[t]{3}{*}{ HSC } & $2_{0,2}-1_{0,1} \quad J=5 / 2-3 / 2 \quad F=2-1$ & 81192.825 & 5.8 & $1.55 \times 10^{-5}$ & 5 & - & - & $-{ }^{a}$ \\
\hline & $2_{0,2}-1_{0,1} \quad J=3 / 2-1 / 2 \quad F=2-1$ & 81194.075 & 5.9 & $1.16 \times 10^{-5}$ & 5 & $+5.39(8)$ & $0.65(15)$ & $4.5(11)$ \\
\hline & $2_{0,2}-1_{0,1} \quad J=5 / 2-3 / 2 \quad F=3-2$ & 81199.988 & 5.9 & $1.55 \times 10^{-5}$ & 7 & $+5.42(5)^{b}$ & $0.43(9)^{b}$ & $4.0(8)^{b}$ \\
\hline
\end{tabular}

Notes. Numbers in parentheses are $1 \sigma$ uncertainties in units of the last digits. The antenna temperature scale can be converted into the main beam brightness temperature by dividing by $\left(B_{\text {eff }} / F_{\text {eff }}\right)$, which is $0.82 / 0.95$ for the IRAM $30 \mathrm{~m}$ telescope at $81 \mathrm{GHz}$. ${ }^{(a)}$ Line is not visible because it overlaps a frequency-switching negative artifact of the HSC $2_{0,2}-1_{0,1} J=5 / 2-3 / 2 F=3-2$ line at $81199.988 \mathrm{MHz}$ (see Fig. 2). ${ }^{(b)}$ Intensity is reduced because the line overlaps a frequency-switching negative artifact of the HSC $2_{0,2}-1_{0,1} J=5 / 2-3 / 2 F=2-1$ line at $81192.825 \mathrm{MHz}$, the line width is likely reduced as well because the line partially overlaps a negative artifact of a line of $\mathrm{CH}_{2} \mathrm{CN}$ lying $7.36 \mathrm{MHz}$ higher in frequency (see Fig. 2), and the derived $V_{\mathrm{LSR}}$ is probably affected as well.

\section{Observations}

The observations were carried out with the IRAM $30 \mathrm{~m}$ telescope in the frame of a $\lambda 3 \mathrm{~mm}$ line survey of L483. We adopted the coordinates of the dense core, $\alpha_{2000.0}=18^{\mathrm{h}} 17^{\mathrm{m}} 29 \mathrm{~s} .8$, $\delta_{2000.0}=-04^{\circ} 39^{\prime} 38^{\prime \prime}$, which correspond to the position of the infrared source IRAS 18148-0440 (Fuller \& Myers 1993). This position also coincides with the intensity maximum of the $\mathrm{CH}_{3} \mathrm{OH}$ emission (Tafalla et al. 2000). We used the EMIR receiver E090 in single-sideband mode, with image rejections $>10 \mathrm{~dB}$, and employed the frequency-switching technique with a frequency throw of 7.2 MHz. We used the FTS backend in its narrow mode, providing a bandwidth of $1.8 \mathrm{GHz}$ and a spectral resolution of $50 \mathrm{kHz}$, which translates into velocity resolutions of $0.19-0.13 \mathrm{~km} \mathrm{~s}^{-1}$ in the $80-116 \mathrm{GHz}$ frequency range. The intensity scale was calibrated using two absorbers at different temperatures and the atmospheric transmission model ATM (Cernicharo 1985; Pardo et al. 2001). We express intensities in terms of $T_{A}^{*}$, the antenna temperature corrected for atmospheric absorption and for antenna ohmic and spillover losses. The uncertainty in $T_{A}^{*}$ is estimated to be around $10 \%$.

The observations around $81 \mathrm{GHz}$, where the lines of HCS and HSC we present here lie, were carried out in two observing sessions, during November 2016 and December 2017. Weather conditions were fairly good during these two observing runs, with amounts of precipitable water vapor in the ranges $2.1-5.8 \mathrm{~mm}$ and $1.5-2.8 \mathrm{~mm}$, respectively, leading to system temperatures of $85-112 \mathrm{~K}$ and $75-92 \mathrm{~K}$, respectively, for elevation angles above $30^{\circ}$. The telescope focus was checked on planets at the beginning of each observing session, which typically lasted $\sim 5 \mathrm{~h}$. The telescope pointing was regularly checked every one hour and half by observing the nearby radio source 1741-038. Pointing errors were typically $2-3^{\prime \prime}$. The beam size of the IRAM $30 \mathrm{~m}$ telescope at $81 \mathrm{GHz}$ is $30^{\prime \prime}$. The on-source integration times were $5.05 \mathrm{~h}$ in November 2016 and $4.12 \mathrm{~h}$ in December 2017. After including data of the two observing sessions and averaging spectra of horizontal and vertical polarizations, the resulting spectrum is very sensitive, with a $T_{A}^{*} \mathrm{rms}$ noise level of $\sim 1.5 \mathrm{mK}$ per $50 \mathrm{kHz}$ channel in the spectral region around $81 \mathrm{GHz}$. The lines of $\mathrm{H}_{2} \mathrm{CS}$ at $101.5 \mathrm{GHz}, 103.0 \mathrm{GHz}$, and $104.6 \mathrm{GHz}$ were observed in May 2017 in the frame of the same $\lambda 3 \mathrm{~mm}$ line survey of L483. Weather conditions were also good, with similar numbers to those given above. More details on these observations will given elsewhere.

\section{Results}

The spectrum of L483 around $80.5 \mathrm{GHz}$ shows a series of emission lines that can be unambiguously assigned to the strongest components of the $2_{0,2}-1_{0,1}$ rotational transition of the radical $\mathrm{HCS}$. In the spectral region around $81.2 \mathrm{GHz}$, the frequencies of two emission lines precisely coincide with two of the strongest components of the $2_{0,2}-1_{0,1}$ rotational transition of the metastable isomer HSC (see Table 1 and Figs. 1 and 2). These species have not been previously observed in space.

The radicals HCS and HSC have a bent structure with a ${ }^{2} A^{\prime}$ ground electronic state. The HSC isomer is $\sim 40 \mathrm{kcal} \mathrm{mol}^{-1}$ less stable than HCS (Puzzarini 2005). Their rotational levels $N_{K_{a}, K_{c}}$ split in a fine (electronic spin-rotation interaction) and hyperfine (H nuclear spin) structure described by the quantum numbers $J$ and $F$, respectively. The rotational spectra of the two radicals have been characterized in the laboratory (Habara et al. 2002; Habara \& Yamamoto 2000), although in the case of the more stable isomer HCS, only transitions with $K_{a}=0$ were observed. Line frequencies were obtained from the Cologne Database for Molecular Spectroscopy ${ }^{1}$ (Müller et al. 2005). The total dipole moments of HCS and HSC have been calculated as $0.96 \mathrm{D}$ and 2.63 D, respectively, with components along the $a$ axis of $0.430 \mathrm{D}$ and $2.493 \mathrm{D}$, respectively (Puzzarini 2005). We use these latter values for the computation of the column densities because the observed lines correspond to $a$-type transitions.

The identification of HCS in L483 relies on the detection of a quintet of lines corresponding to the five strongest fine and hyperfine components of the $2_{0,2}-1_{0,1}$ rotational transition. The line parameters are given in Table 1 and the lines are shown in Fig. 1. These five transitions have line strengths that decrease with increasing frequency (see Einstein coefficients in Table 1), which is consistent with the observed pattern of line intensities. The brightest line, lying at $80553.516 \mathrm{MHz}$, has a peak intensity of $T_{A}^{*} \sim 15 \mathrm{mK}$ and is well detected above the noise level, with a signal-to-noise ratio $(S / N) \gtrsim 10 \sigma$, while the faintest line, lying at $80618.820 \mathrm{MHz}$, is observed with a peak intensity of $T_{A}^{*} \sim 5 \mathrm{mK}$ and is detected at a lower confidence level, $\sim 3 \sigma$ (see Fig. 1). In the case of the metastable isomer HSC, the $2_{0,2}-1_{0,1}$ rotational transition also has multiple components arising from the fine and hyperfine structure. The three strongest components are listed in Table 1. Two components are clearly detected in the spectrum of L483 at confidence levels of $4-5 \sigma$, although the component

1 See http://www .astro. uni-koeln.de/cdms/ 


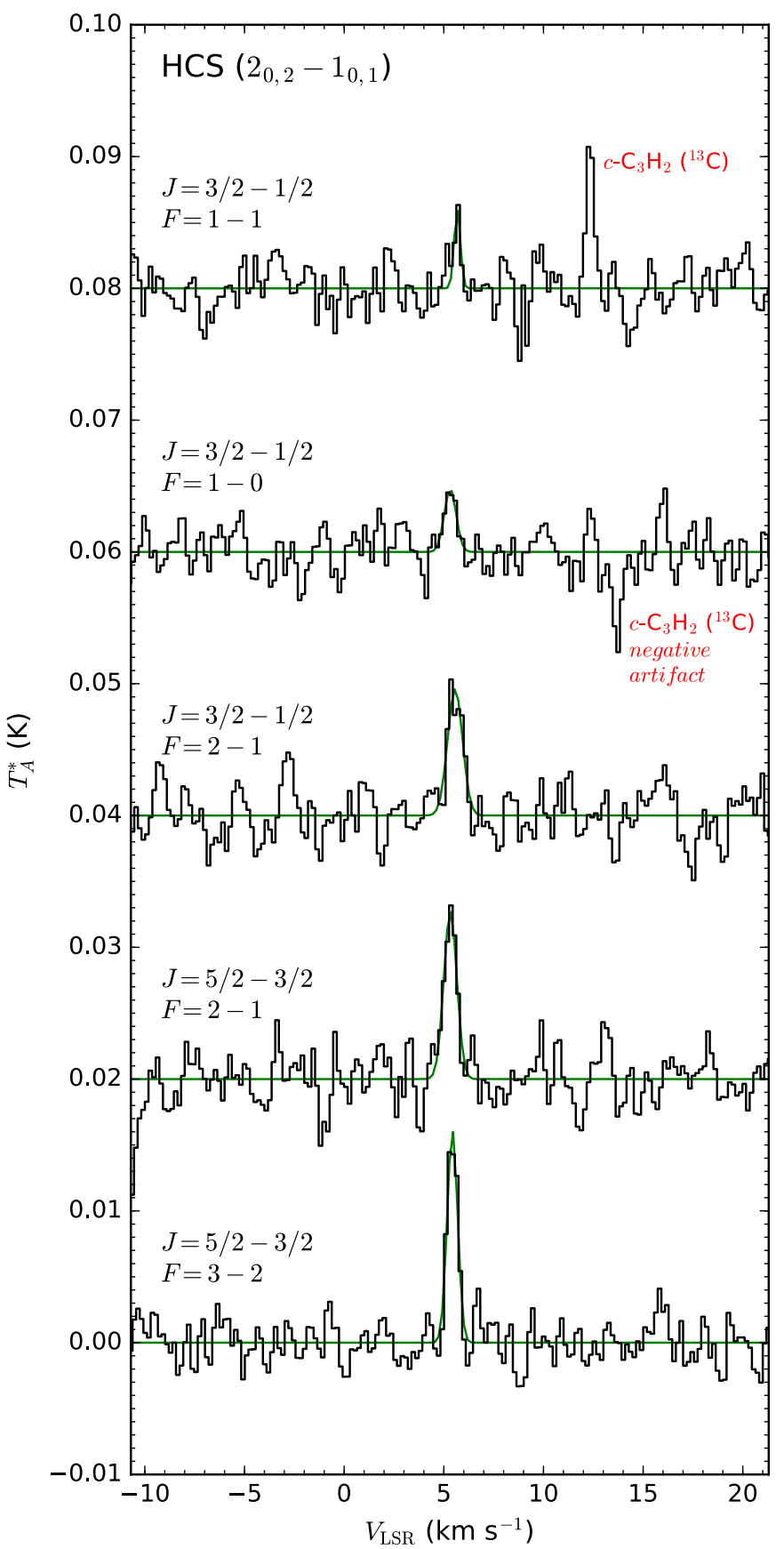

Fig. 1. Lines of HCS observed in L483 are shown from bottom to top in order of increasing frequency. Note that the observed line intensity decreases as the frequency increases.

lying at $81192.825 \mathrm{MHz}$ is not visible (see Fig. 2). The most likely reason is that the frequency separation between the components at $81192.825 \mathrm{MHz}$ and $81199.988 \mathrm{MHz}$ is similar to the frequency throw of $7.2 \mathrm{MHz}$ adopted in the frequency-switching observations. Therefore, it accidentally happens that each line overlaps with a frequency-switching negative artifact of the other line. As a result, the weaker line at $81192.825 \mathrm{MHz}$ in our observed spectrum is artificially cancelled and the intensity of the stronger line at $81199.988 \mathrm{MHz}$ is also reduced.

Assuming a rotational temperature of $10 \mathrm{~K}$, which is close to the gas kinetic temperature (Fuller \& Myers 1993; Anglada et al. 1997), we derive beam-averaged column densities in L483 of $7 \times$ $10^{12} \mathrm{~cm}^{-2}$ and $1.8 \times 10^{11} \mathrm{~cm}^{-2}$ for HCS and HSC, respectively. These values translate into fractional abundances relative to $\mathrm{H}_{2}$

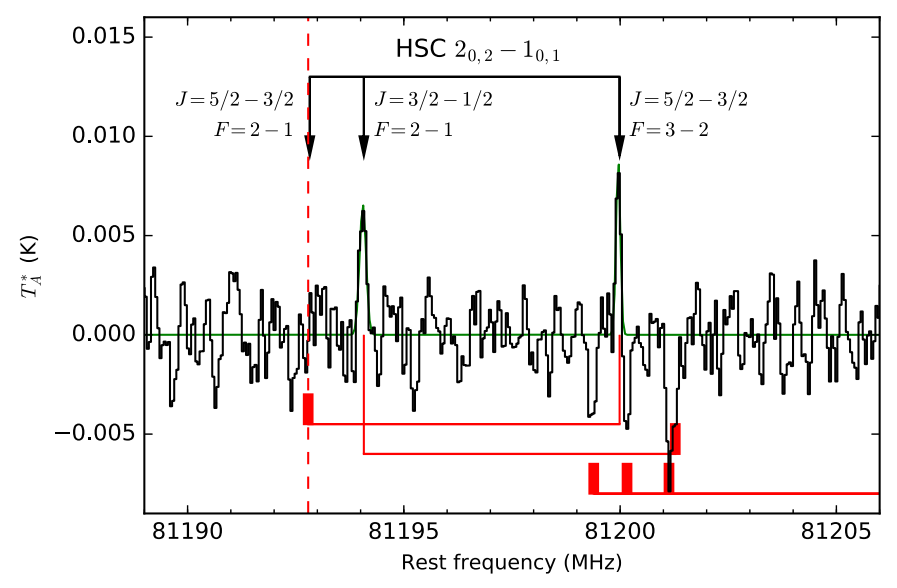

Fig. 2. Spectra of L483 around $81.2 \mathrm{GHz}$ showing the lines assigned to HSC. The rest frequency scale corresponds to an LSR systemic velocity of $+5.3 \mathrm{~km} \mathrm{~s}^{-1}$. The bottom vertical red marks indicate the position of frequency-switching negative artifacts. Note that the $J=5 / 2-3 / 2$ $F=2-1$ line is not visible because it is artificially cancelled with a frequency-switching negative artifact, while the $J=5 / 2-3 / 2 F=3-2$ is also affected by negative artifacts (see text).

of $2 \times 10^{-10}$ for HCS and $6 \times 10^{-12}$ for HSC, adopting the value of $N\left(\mathrm{H}_{2}\right)$ of $3 \times 10^{22} \mathrm{~cm}^{-2}$ derived by Tafalla et al. (2000) from observations of $\mathrm{C}^{17} \mathrm{O}$. We note that the column density of HCS only includes $K_{a}=0$ levels. Using the rotational constants A, B, and $\mathrm{C}$ from Habara et al. (2002), we estimate that the contribution of $K_{a} \neq 0$ levels to the partition function at $10 \mathrm{~K}$ is just $2 \%$. Therefore, the HCS/HSC column density ratio in L483 is $\sim 40$. The related molecule $\mathrm{H}_{2} \mathrm{CS}$ was also observed in the course of the $\lambda 3 \mathrm{~mm}$ line survey of L483. For $\mathrm{H}_{2} \mathrm{CS}$ we estimate excitation temperatures in the range 5-10 K, adopting a volume density of $\mathrm{H}_{2}$ of $3.4 \times 10^{4} \mathrm{~cm}^{-3}$ (Jørgensen et al. 2002) and taking into account that $\mathrm{H}_{2} \mathrm{CS}$ should be more efficiently excited through collisions than $\mathrm{H}_{2} \mathrm{CO}$ because of the larger geometrical cross section. From the observed intensities and assuming rotational temperatures in the range $5-10 \mathrm{~K}$, we derive a beam-averaged column density of $(1.0-2.1) \times 10^{13} \mathrm{~cm}^{-2}$ for $\mathrm{H}_{2} \mathrm{CS}$.

Based on this, the radical HCS has an abundance similar to that of thioformaldehyde, with a $\mathrm{H}_{2} \mathrm{CS} / \mathrm{HCS}$ column density ratio of $1.4-3$, that is to say, on the order of unity. The situation here is markedly different from that of the oxygen-bearing analogs. In L483, the $\mathrm{H}_{2} \mathrm{CO} / \mathrm{HCO}$ column density ratio is 19 (Tafalla et al. 2000; Agúndez et al. 2015a), in line with the values found in other cold dark clouds, which are in the range 7-22 (see Ocaña et al. 2017 and references therein). Therefore, while the radical HCO is around ten times less abundant than $\mathrm{H}_{2} \mathrm{CO}$ in cold dark clouds, in L483 the HCS radical is as abundant as $\mathrm{H}_{2} \mathrm{CS}$. Moreover, HCS is even more abundant than $\mathrm{HCO}$ in L483. The presence of the metastable isomer HSC in cold dark clouds also makes a difference between oxygen and sulfur chemistries. The oxygen analog HOC has not been observed in space, essentially because there is a complete lack of experimental information for this species. The two metastable isomers HOC and HSC both lie $\sim 40 \mathrm{kcal} \mathrm{mol}^{-1}$ above their respective stable isomers, HCO and HCS. However, HOC also lies above the fragments $\mathrm{H}$ and $\mathrm{CO}$, whereas $\mathrm{HSC}$ is more stable than $\mathrm{H}$ and CS (Marenich \& Boggs 2003; Puzzarini 2005; Pérez-Juste \& Carballeira 2007). Still, the metastable isomer HOC should be stable enough to be characterized because the decay to $\mathrm{H}$ and CO is predicted to have a barrier (Marenich \& Boggs 2003). The search for HOC in space must in any case await its detection in the laboratory. 


\section{Discussion}

The fractional abundances relative to $\mathrm{H}_{2}$ derived for HCS and HSC, a few $10^{-10}$ and several $10^{-12}$, respectively, indicate that these species do not lock an important fraction of sulfur. However, the finding that HCS has an abundance similar to $\mathrm{H}_{2} \mathrm{CS}$ and the detection of the metastable isomer HSC place interesting constraints on the chemistry of sulfur in cold dark clouds.

The chemistry of sulfur in dark clouds has recently been revisited by Vidal et al. (2017) in a study in which the sulfur chemical network was thoroughly revised. Interestingly, this study discussed the potential detectability of HCS based on the non-negligible abundance predicted for this radical. The calculated abundance between $10^{5}$ and $10^{6} \mathrm{yr}$ is in the range $10^{-12}-10^{-11}$ relative to $\mathrm{H}_{2}$, however, which is below the observed value by at least one order of magnitude. Moreover, the observational finding of an $\mathrm{H}_{2} \mathrm{CS} / \mathrm{HCS}$ abundance ratio close to 1 is not well accounted for by their model, which predicts that $\mathrm{H}_{2} \mathrm{CS}$ is one to two orders of magnitude more abundant than HCS. No prediction is made for the metastable isomer HSC as this species is not included in their model.

The main reactions leading to $\mathrm{HCS}$ in the model of Vidal et al. (2017) are

$\mathrm{S}+\mathrm{CH}_{2} \rightarrow \mathrm{HCS}+\mathrm{H}$,

$\mathrm{H}_{2} \mathrm{CS}^{+}+\mathrm{e}^{-} \rightarrow \mathrm{HCS}+\mathrm{H}$,

$\mathrm{H}_{3} \mathrm{CS}^{+}+\mathrm{e}^{-} \rightarrow \mathrm{HCS}+\mathrm{H}+\mathrm{H}$,

$\mathrm{C}+\mathrm{H}_{2} \mathrm{~S} \rightarrow \mathrm{HCS}+\mathrm{H}$.

Reactions (1)-(3) are the sulfur analogs of the main reactions forming the $\mathrm{HCO}$ radical in cold dark clouds, in which case chemical models calculate an abundance in good agreement with observed values (Agúndez et al. 2015a; Bacmann \& Faure 2016; Ocaña et al. 2017). Observations indicate, however, that the oxygen and sulfur cases behave differently, as indicated by the different abundance ratios: $\mathrm{H}_{2} \mathrm{CO} / \mathrm{HCO}>1$ and $\mathrm{H}_{2} \mathrm{CS} / \mathrm{HCS} \sim 1$. Reaction (1) is basically considered by similarity with the $\mathrm{O}+\mathrm{CH}_{2}$ reaction, which has been suggested to yield HCO. We note, however, that it is not fully clear whether this reaction can proceed (Ocaña et al. 2017), and thus it would be of great interest to study the reactions of $\mathrm{O}$ and $\mathrm{S}$ atoms with $\mathrm{CH}_{2}$ theoretically to evaluate their role in the formation of $\mathrm{HCO}$ and HCS, respectively, and of their metastable isomers HOC and HSC. Reaction (2) is a feasible route to HCS, although since the most stable structure of the ion $\mathrm{H}_{2} \mathrm{CS}^{+}$has the two hydrogen atoms bonded to carbon (Curtis et al. 1992), it would require a considerable rearrangement to yield HSC. Reaction (3) is a likely pathway to HCS. Moreover, the similar abundances derived for $\mathrm{H}_{2} \mathrm{CS}$ and $\mathrm{HCS}$ are suggestive of a common origin, and thus the dissociative recombination of $\mathrm{H}_{3} \mathrm{CS}^{+}$could be a common source of both species. Reaction (4) has been studied experimentally and theoretically and offers an interesting route to both HCS and HSC. The reaction is rapid at room temperature, with a measured rate constant of $2.5 \times 10^{-10} \mathrm{~cm}^{3} \mathrm{~s}^{-1}$ (Deeyamulla \& Husain 2006). Theoretical calculations indicate that both HCS and HSC can form without energy barrier (Ochsenfeld et al. 1999; Galland et al. 2001), although the formation of HCS is more exothermic (40-44 kcal mol${ }^{-1}$ ) than that of HSC (just 2-4 $\mathrm{kcal} \mathrm{mol}^{-1}$ ), which could favor HCS over HSC. This would be in agreement with crossed-beam experiments (Kaiser et al. 1999; Ochsenfeld et al. 1999). We note that although reactions (1)-(4) are plausible routes to HCS, their implementation in the model of Vidal et al. (2017) is not enough to increase the abundance of this radical up to the level observed in L483. It is clear that the chemistry of sulfur still needs to be revised in the light of the new observational constraints provided by the detection of HCS and HSC.

\section{Conclusions}

We have presented the first detection in space of the thioformyl radical (HCS) and its metastable isomer HSC. These species have been observed in the molecular cloud L483, with fractional abundances relative to $\mathrm{H}_{2}$ of a few $10^{-10}$ for HCS and various $10^{-12}$ for HSC. The fraction of sulfur locked by these radicals is low. However, their detection places interesting constraints on the chemistry of sulfur in dark clouds. We find significant differences with respect to the oxygen analog species. First, the observed $\mathrm{H}_{2} \mathrm{CS} / \mathrm{HCS}$ abundance ratio is close to unity, while the $\mathrm{H}_{2} \mathrm{CO} / \mathrm{HCO}$ abundance ratio in dark clouds is found to be around 10. Second, the metastable isomer HSC is found with a low abundance, while its oxygen analog $\mathrm{HOC}$ has not yet been observed in space. The latest chemical models of dark clouds cannot account for the relative abundance of $\mathrm{HCS}$, the $\mathrm{H}_{2} \mathrm{CS} / \mathrm{HCS}$ ratio, and the presence of HSC, suggesting that a revision of the chemistry of sulfur is required.

Acknowledgements. We thank the referee for a careful reading of the manuscript and the IRAM $30 \mathrm{~m}$ staff for their help during the observations. We acknowledge funding support from the European Research Council (ERC Grant 610256: NANOCOSMOS) and from Spanish MINECO through grant AYA2016-75066C2-1-P. M.A. also acknowledges funding support from the Ramón y Cajal programme of Spanish MINECO (RyC-2014-16277).

\section{References}

Adande, G. R., Halfen, D. T., Ziurys, L. M., et al. 2010, ApJ, 725, 561 Agúndez, M., \& Wakelam, V. 2013, Chem. Rev., 113, 8710

Agúndez, M., Cernicharo, J., \& Guélin, M. 2015a, A\&A, 577, L5 Agúndez, M., de Vicente, P., et al. 2015b, A\&A, 579, L10 Anglada, G., Sepúlveda, I., \& Gómez, J. F. 1997, A\&AS, 121, 255 Bacmann, A., \& Faure, A. 2016, A\&A, 587, A130 Boogert, A. C. A., Schutte, W. A., Helmich, F. P., et al. 1997, A\&A, 317, 929 Cernicharo, J. 1985, IRAM Internal Report, 52

Cernicharo, J., Lefloch, B., Agúndez, M., et al. 2018, ApJ, 853, L22 Curtiss, L. A., Nobes, R. H., Pople, J. A., \& Radom, L. 1992, J. Chem. Phys., 97, 6766

Deeyamulla, M. P., \& Husain, D. 2006, J. Photochem. Photobiol. A: Chem., 184, 347

Druard, C., \& Wakelam, V. 2012, MNRAS, 426, 354

Fuente, A., Cernicharo, J., Roueff, E., et al. 2016a, A\&A, 593, A94

Fuente, A., Goicoechea, J. R., Pety, J., et al. 2016b, ApJ, 851, L49

Fuller, G. A., \& Myers, P. C. 1993, ApJ, 418, 273

Galland, N., Caralp, F., Rayez, M.-T., et al. 2001, J. Phys. Chem. A, 105, 9893

Goicoechea, J. R., Pety, J., Gerin, M., et al. 2006, A\&A, 456, 565

Habara, H., \& Yamamoto, S. 2000, J. Chem. Phys., 112, 10905

Habara, H., Yamamoto, S., \& Amano, T. 2002, J. Chem. Phys., 116, 9232

Holdship, J., Viti, S., Jiménez-Serra, I., et al. 2016, MNRAS, 463, 802

Jenkins, E. B. 2009, ApJ, 700, 1299

Jiménez-Escobar, A., \& Muñoz Caro, G. M. 2011, A\&A, 536, A91

Jørgensen, J. K., Schöier, F. L., \& van Dishoeck, E. F. 2002, A\&AS, 389, 908

Kaiser, R. I., Ochsenfeld, C., Head-Gordon, M., \& Lee, Y. T. 1999, J. Chem. Phys., 110, 2391

Marenich, A. V., \& Boggs, J. E. 2003, J. Phys. Chem. A, 107, 2343

Martín-Doménech, R., Jiménez-Serra, I., Muñoz Caro, G. M., et al. 2016, A\&A, 585, A112

Müller, H. S. P., Schlöder, F., Stutzki, J., \& Winnewisser, G. 2005, J. Mol. Struct., 742,215

Ocaña, A. J., Jiménez, E., Ballesteros, B., et al. 2017, ApJ, 850, 28

Ochsenfeld, C., Kaiser, R. I., Lee, Y. T., \& Head-Gordon, M. 1999, J. Chem. Phys., 110, 9982

Palumbo, M. E., Geballe, T. R., \& Tielens, A. G. G. M. 1997, ApJ, 479, 839

Pardo, J. R., Cernicharo, J., \& Serabyn, E. 2001, IEEE Trans. Antennas Propag., 49, 1683

Pérez-Juste, I., \& Carballeira, L. 2007, J. Chem. Phys., 127, 164303

Puzzarini, C. 2005, J. Chem. Phys., 123, 024313

Tafalla, M., Myers, P. C., Mardones, D., \& Bachiller, R. 2000, A\&A, 359, 967

Vidal, T. H. G., Loison, J.-C., Yassin Jaziri, A., et al. 2017, MNRAS, 469, 435 